\title{
Editorial
}

\section{Advances in Electrocardiogram Signal Processing and Analysis}

\author{
William Sandham, ${ }^{1}$ David Hamilton, ${ }^{2}$ Pablo Laguna, ${ }^{3}$ and Maurice Cohen ${ }^{4}$ \\ ${ }^{1}$ Scotsig, Glasgow G12 9PF, UK \\ ${ }^{2}$ Ateeda Limited, Edinburgh EH3 8EG, UK \\ ${ }^{3}$ Department of Electronic Engineering and Communications, Zaragoza University, 50018 Zaragoza, Spain \\ ${ }^{4}$ UCSF Fresno Center for Medical Education, School of Medicine, University of California, San Francisco, Fresno, \\ CA 93701-2302, USA
}

Received 3 April 2007; Accepted 3 April 2007

Copyright (c) 2007 William Sandham et al. This is an open access article distributed under the Creative Commons Attribution License, which permits unrestricted use, distribution, and reproduction in any medium, provided the original work is properly cited.

Since itsinvention by the Dutchman Willem Einthoven (1860-1927) during the late 19th and early 20th centuries, when it was little more than a scientific curiosity, the electrocardiogram (ECG) has developed into one of the most important and widely used quantitative diagnostic tools in medicine. It is essential for the identification of disorders of the cardiac rhythm, extremely useful for the diagnosis and management of heart abnormalities such as myocardial infarction (heart attack), and it offers helpful clues to the presence of generalized disorders that affect the rest of the body, such as electrolyte disturbances and drug intoxication.

Recording and analysis of the ECG now involve a considerable amount of signal processing; for $\mathrm{S} / \mathrm{N}$ enhancement, beat detection and delineation, automated classification, compression, hidden information extraction, and dynamic modeling. These involve a whole variety of innovative signal processing methods, including adaptive techniques, time-frequency and time-scale procedures, artificial neural networks and fuzzy logic, higher-order statistics and nonlinear schemes, fractals, hierarchical trees, Bayesian approaches, and parametric models, amongst others.

This special issue reviews the current status of ECG signal processing and analysis, with particular regard to recent innovations. It reports major achievements by academic and commercial research institutions and individuals, and provides an insight into future developments within this exciting and challenging area. It is perhaps appropriate that a special issue of EURASIP JASP be devoted to ECG signal processing and analysis, since the ECG is now celebrating its centennial (Dijk and Van Loon [1]).

The first paper, "Multiadaptive bionic wavelet transform: application to ECG denoising and baseline wandering reduction," by O. Sayadi and M. B. Shamsollahi, describes a new modified wavelet transform that can be used to remove a wide range of noise from an ECG signal. Signal decomposition is obtained using the bionic wavelet transform, adaptively determining both the center frequency of each scale, together with the $T$-function. A threshold rule is then applied. The method was tested with both real and simulated ECG signals. Results demonstrate a significantly better noise reduction compared with standard wavelet transform techniques; the average signal-to-noise ratio improved by a factor of 1.82 (best case). The method also produced better results in relation to baseline wandering calculations for both DC components and shifts.

The second paper, "Hardware implementation of a modified delay-coordinate mapping-based QRS complex detection algorithm," by Matej Cvikl et al., describes a QRS detection algorithm specifically designed for hardware implementation. It uses a modified delay-coordinate mappingbased algorithm, prefiltering, and better threshold calculation methods to improve the original two-dimensional phase-space portrait. Results on the MIT-BIH arrhythmia and long-term ST databases indicate excellent sensitivity and predictivity.

The next two papers cover the important topic of ECG compression. "A simple method for guaranteeing ECG quality in real-time wavelet lossy coding," by A. Alesanco and J. García, proposes a new distortion index wavelet-weighted PRD (WWPRD), which aims to provide a more realistic description of the clinical distortion of the compressed signal. The method applies the wavelet transform and the subsequent coding uses the set partitioning in hierarchical trees (SPIHT) algorithm. By thresholding the WWPRD in the wavelet transform domain, a very precise reconstruction error was achieved, allowing clinically useful 
reconstructed signals to be obtained. Again, results from two ECG databases indicate that the method can accurately control the quality in terms of mean value with a low standard deviation. Some discussion of the impact of baseline wander and noise is included, along with a description of the clinical validation sought for these results. The second paper in this category, "Lossless compression schemes for ECG signals using neural network predictors," by K. Ramakrishnan and E. Chikkannan, presents lossless compression schemes for ECG signals based on neural network predictors and entropy encoders. Decorrelation is achieved by nonlinear prediction in the first stage and encoding of the residues is done by using lossless entropy encoders in the second stage. Different types of lossless encoders, such as Huffman, arithmetic, and runlength encoders are used. The performances of the proposed neural network predictor-based compression schemes are evaluated using standard distortion and compression efficiency measures. Selected records from the MIT$\mathrm{BIH}$ arrhythmia database are used for performance evaluation. The proposed compression schemes are compared with linear predictor-based compression schemes and it is shown that about $11 \%$ improvement in compression efficiency can be achieved for neural network predictor-based schemes with the same quality and similar setup. They are also compared with other known ECG compression methods and the experimental results show that superior performances in terms of the distortion parameters of the reconstructed signals can be achieved with the proposed schemes.

Segmentation and delineation is the topic covered by the fifth paper, "Combining wavelet transform and hidden Markov models for ECG segmentation," by R. V. Andreão and J. Boudy. This work aims at providing new insights into the ECG segmentation problem using wavelets. The wavelet transform has been originally combined with a hidden Markov model (HMM) framework in order to carry out beat segmentation and classification. A group of five continuous wavelet functions commonly used in ECG analysis has been implemented and compared using the same framework. All experiments were realized on the QT database, which is composed of a representative number of ambulatory recordings of several individuals and is supplied with manual labels made by a physician. A consistent set of experiments was performed, and comparable results with other published works were obtained in terms of beat delineation and premature ventricular beat (PVC) detection, independently of the type of wavelet. Optimum performance was achieved by combining two wavelet functions in the delineation stage.

The next two papers address the topic of ECG modeling. In the first paper, "Modeling of electrocardiogram signals using predefined signature and envelope vector sets," by Hakan Gürkan et al., a method for modeling ECG signals is proposed based on a predefined signature and envelope vector set (PSEVS). The ECG signal is reconstructed by multiplying three parameters: predefined signature vector $\left(\mathrm{PSV}_{R}\right)$, predefined vector envelope $\left(\mathrm{PEV}_{K}\right)$, and frame-scaling coefficient (FCS). The first two measures are labeled and stored to describe the signal in the reconstruction process. An ECG signal frame is modeled as members of the sets labeled $R$ and $K$ and the frame-scaling coefficient in the least-mean-square sense. The method is assessed using the percentage of root-meansquare difference and also using visual inspection measures. Results show that the method provides significant data compressions ratios and low-level root-mean-square differences while preserving diagnostic information, thus significantly reducing the bandwidth required for telediagnosis. The second paper in this category, "multi-channel ECG and noise modeling: application to maternal and fetal ECG signals," by Reza Sameni et al., presents a three-dimensional dynamic model of the electrical activity of the heart. It is based on the single dipole model of the heart and is later related to the body surface potentials through a linear model which accounts for the temporal movements and rotations of the cardiac dipole, together with a realistic ECG noise model. The proposed model is also generalized to maternal and fetal ECG mixtures recorded from the abdomen of pregnant women in single and multiple pregnancies. The applicability of the model for the evaluation of signal processing algorithms is illustrated using independent component analysis. Considering the difficulties and limitations of recording long-term ECG data, especially from pregnant women, the model described in this paper may serve as an effective means of simulation and analysis of a wide range of ECGs, including adults and fetuses.

The next two papers address a very useful DSP technique for ECG signals-principal component analysis (PCA). The first paper, "Principal component analysis in ECG signal processing," by Francisco Castells et al., provides a comprehensive review of the topic, and covers the fundamentals of PCA and its relationship to the Karhunen-Loève transform. Aspects on PCA related to data with temporal and spatial correlations are considered, together with adaptive estimation of principal components. Several ECG applications are reviewed, particularly where PCA techniques have been successfully employed. These include data compression, ST-T segment analysis for the detection of myocardial ischemia and abnormalities in ventricular repolarization, extraction of atrial fibrillatory waves for detailed characterization of atrial fibrillation, and analysis of body surface potential maps. The second paper, "A principal component regression approach for estimating ventricular repolarization duration variability," by Mika Tarvainen et al., introduces the idea of computing the ventricular repolarization duration (VRD) variability, not from some fiducial point (typically $R$-wave to $T$ peak or $T$-end) which has a large delineation uncertainty, but from a PCA decomposition parameter. PCA decomposition is applied to $T$-wave segments synchronized with the $R$-wave. The first eigenvalue is considered to be representative of the mean $T$-wave shape and the second eigenvalue is of the $T$ variability. Robustness is presented as the major advantage of the method, and good correlation results with classical variability measures are demonstrated. Results are presented on a stress test application.

The final selection of papers deal with the analysis of the ECG for detecting specific medical conditions. The first paper, "Diurnal changes of heart rate and sympathovagal activity for temporal patterns of transient ischemic episodes 
in 24-hour electrocardiograms," by A. Smrdel and F. Jager, presents a method for analyzing temporal patterns of transient ST segment changes compatible with ischemia, with the hypothesis that different patterns are a result of different physiological mechanisms. The study is based on 24-hour records that were divided into morning, day, and night intervals. Three temporal patterns are considered: salvo, periodic, and sporadic. Both time and frequency measures of heart rate in the neighborhood of ischemic events were analyzed using an adaptive autoregressive method with a recursive least-square algorithm, for consistent spectral tracking of heart rate, to study frequency-domain sympathovagal behavior during ischemia. Results indicate two distinct populations that differ according to mechanism and temporal patterns of ischemia. In the second paper, "Real-time cardiac arrhythmia detection using WOLA filterbank analysis of EGM signals," by Hamid Sheikhzadeh et al., novel methods of cardiac rhythm detection are proposed that are based on time-frequency analysis by a weighted overlap-add (WOLA) oversampled filterbank. Cardiac signals are obtained from intracardiac electrograms and are decomposed into the timefrequency domain and are analyzed by parallel peak detectors in selected frequency subbands. The coherence (synchrony) of the subband peaks is analyzed and employed to detect an optimal peak sequence representing the beat locations. By further analysis of the synchrony of the subband beats and the periodicity and regularity of the optimal beat, various possible cardiac events (including fibrillation, flutter, and tachycardia) are detected. Evaluation results show very good performance in clean and noisy conditions, and robustness to far-field $R$-wave interference. The third paper, "Corrected integral shape averaging applied to obstructive sleep apnea detection from the electrocardiogram," by S. Boudaoud et al., presents a technique called corrected integral shape averaging (CISA) for quantifying shape and shape differences in a set of signals. The method can be used to account for signal differences which are purely due to affine time warping (jitter and dilation/compression), and hence provides access to intrinsic shape fluctuations. CISA can also be used to define a distance between shapes which have useful mathematical properties, and the procedure also allows joint estimation of the affine time parameters. Numerical simulations are presented to support the algorithm. CISA provides a well-defined shape distance, which can be used in shape clustering applications based on distance measures such as $k$-means. An application is presented in which CISA shape clustering is applied to $P$-waves extracted from the ECGs of subjects suffering from sleep apnea. The resulting shape clustering distinguishes ECG segments recorded during apnea from those recorded during normal breathing with a sensitivity of $81 \%$ and specificity of $84 \%$. The fourth paper, "Time-frequency analysis of heart rate variability for neonatal seizure detection,” by M. B. Malarvili et al., proposes a technique for detecting epileptic seizures from the ECG in the human neonate. The suitability of heart rate variability (HRV) as a tool for seizure detection in newborns is explored. Features of HRV in different frequency bands have been obtained by means of quadratic time-frequency dis- tributions (TFDs). The first conditional moment of HRV, which is the mean/central frequency in the LF band (0.03$0.07 \mathrm{~Hz})$ and the variance in the HF band $(0.15-0.6 \mathrm{~Hz})$ is shown to have the ability to discriminate the newborn seizure from nonseizure. In the final paper in this category, "Clustering and symbolic analysis of cardiovascular signals: discovery and visualization of medically relevant patterns in long-term data with limited prior knowledge," by Zeeshan Syed et al., automated techniques are presented for analyzing large amounts of cardiovascular data without the requirement of a priori knowledge of disease states. The process begins by transforming continuous waveform signals into symbolic strings derived from the data. Morphological features are used to partition heartbeats into clusters by maximizing the dynamic time-warped sequence-aligned separation of clusters. Each cluster is then assigned a symbol, and the original signal is replaced by the corresponding sequence of symbols, thus greatly reducing the amount of data. The sequence analysis is used to discover rhythms, transient patterns, abnormal changes in entropy, and clinically significant relationships among multiple streams of physiological data. The method was tested on cardiologist-annotated ECG data for 48 patients. The labeling process for heartbeats showed 98.6\% agreement with the assessment of the cardiologist, and often provided finer-grain distinctions. Using no prior knowledge, cases with a number of different arrhythmias were successfully detected.

\section{ACKNOWLEDGMENTS}

We are extremely grateful to all the reviewers who took time and consideration to assess the submitted manuscripts. Their diligence has contributed greatly to ensuring that final papers have conformed to the high standards expected in this publication. Finally, we wish to dedicate this special issue to the memory of Professor Arnon Cohen, Ben Gurion University, Israel, who passed away in 2004, and who was one of the major pioneers in in ECG signal processing and analysis. A brief biography of Professor Cohen appears below. We are indebted to his family, friends, and professional colleagues for providing this information.

\section{Professor Arnon Cohen (1938-2005)}

Arnon Cohen was born in Haifa, Israel, in 1938. He received the B.S. and M.S. degrees in electrical engineering from the Technion - Israel Institute of Technology, in 1964 and 1966, respectively, and the Ph.D. degree in electrical and biomedical engineering, from Carnegie-Mellon University in 1970. From 1970 to

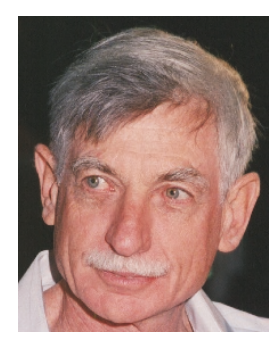
1972, he was an Assistant Professor of electrical and biomedical engineering in the University of Connecticut. In 1972, he returned with his family to Israel, and joined the newly formed Department of Electrical and Computer Engineering, and the Biomedical Engineering Program, in the Ben Gurion University of the Negev, at Beer Sheva, Israel. The 
Electrical Engineering Department at Ben Gurion University was first established in the late sixties as an extension of the Technion - Israel Institute of Technology, and Technion professors used to fly from Haifa to Beer Sheva, perform their teaching duties, and fly back home. Beer Sheva was relatively remote from the center of Israel, and joining the new university in the Negev was considered then to be a pioneering adventure. In 1973, Arnon was appointed as an Associate Professor.

From 1974 to 1976 and from 1978 to 1986 , Arnon was Chairman of the University's Biomedical Engineering Program, and from 1976 to 1977, he was a Visiting Professor at the Electrical Engineering Department, Colorado State University. In 1986 he was promoted to Full Professor of electrical, computer and biomedical engineering. During 19891990, he spent a year in Cape Town, South Africa, as a Visiting Scientist at the South African Medical Research Council (MRC), the Foundation of Research and Development (FRD), and the University of Cape Town. In addition to his academic duties, Arnon served as a consultant to several Israeli hi-tech companies (1982-2000), and held the position of CTO at Sesame Systems (1985-1986) and the DSP Group (1997-2000).

Arnon was a Senior Member of IEEE, and a Member of the Israeli Society for Medical and Biomedical Engineering (IFMBE affiliated). He was the Associate Editor of the IEEE Transactions on Biomedical Engineering (19962000), Guest Editor of the IEEE-EMBS Magazine (Special Issue on Biomedical Signal Databases), and Chairman of the IEEE Biomedical Engineering Society, Israeli Chapter (19962001).

Arnon's research interests covered many areas in signal processing and recognition, mainly in biomedical and speech applications. Ten Ph.D. students and more than thirty M.S. students completed their degrees under his supervision. Many of them have now taken senior research and development positions in Israeli hi-tech companies, ensuring that his legacy is kept alive.

His research in acoustic signal processing as a medical tool was creative and original, and included extensive collaboration with medical doctors at the Soroka Medical Center in Beer Sheva. He made some significant contributions to the analysis and compression of ECG signals, and to several other applications involving biomedical signal processing (EEG, EMG, breathing and stridor sounds, lung sounds during incubation, infant's cry, snoring signals, and more).

Arnon collaborated with researchers in Ben Gurion University as well as in other universities on language-related issues and audio analysis and recognition, and was an inspirational leader within these groups. He focused on issues of speech (phoneme, vowel, and word) recognition, speaker recognition, and speaker verification. He was a wellrespected author and published more than 100 research papers in refereed international journals and conference proceedings. He also wrote the seminal book Biomedical Signal Processing, (CRC Press, Boca Raton, Fla, 1986).

Arnon was diagnosed with Leukemia in 1999, not long after his beloved wife Yama (Miriam) passed away. He offi- cially retired in 2003, but continued working with his graduate students until his final days. He passed away in February 2005 at the age of 67, after a sharp downturn in his condition. However, he is survived by three sons (Boaz, Gilad, and Nadav), all electrical engineers, a daughter-in-law (Michal), and three grandchildren (Shira, Jonathan, and Itay), to whom he showed great love and affection. A more recent addition to the family, granddaughter (Hagar) will only know her grandfather through tales and anecdotes, of which there are many.

In his spare time, Arnon enjoyed traveling, hiking, wildflower photography, and listening to classical music and opera. His death is a sad loss to everyone who were fortunate enough to know him, and he will be greatly missed by his family, friends, students, and colleagues.

\section{William Sandham David Hamilton Pablo Laguna Maurice Cohen}

\section{REFERENCES}

[1] J. Dijk and B. Van Loon, "The electrocardiogram centennial: Willem Einthoven (1860-1927)," Proceedings of the IEEE, vol. 94, no. 12, pp. 2182-2185, 2006.

William Sandham is Managing Director of Scotsig, an independent signal and image processing research, consultancy, and training company based in Glasgow, and he is a Visiting Professor in the Department of Bioengineering, University of Strathclyde. $\mathrm{He}$ received the B.S. and Ph.D. degrees from the Universities of Glasgow (1974) and Birmingham (1981), worked as a Medical Physicist from 1974 to 1976, and was a Geo-

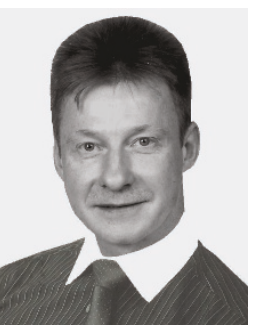

physicist with the British National Oil Corporation and Britoil, from 1980 to 1986. From 1986 to 2003, he was a Lecturer/Senior Lecturer/Reader at the University of Strathclyde, Glasgow. He has published over 150 technical papers and 5 books, he is a Senior Member of the IEEE, and Member of the EAGE and SEG. He has served on a number of IEEE and other editorial boards, and the conference organizing committees of ICASSP (1989), TA-91 (1991), and EUSIPCO (1994). He was Chairman of IEEE and EAGE International Workshops in Glasgow (1991) and Geneva (1997), the IET MEDSIP Conference (2006), and is Chairman of the IET Symposium on Technology in Diabetes Care (2007). He has been an Invited Lecturer at a number of leading research institutions across the UK, Europe, and South America, and has acted as a Consultant for major companies in Europe and North America.

David Hamilton is a Ph.D. MIET Chartered Electrical Engineer. Since February 2007, he has been Chief Executive Officer for Ateeda Limited, a semiconductor software company, he has worked as Senior Lecturer in the Department of Electronic and Electrical Engineering at the University of Strathclyde since January 1991. He has wide interests in a variety of fields. These range from biomedical to seismic surveying,

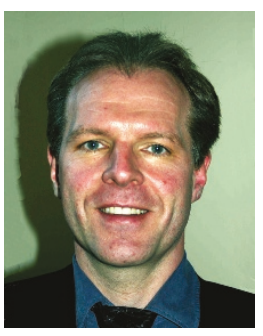


often with a strong signal processing theme. Biomedical interests include ECGs (acquisition hardware through the signal processing applied to ECGs) through EEGs (brain-computer interface) and ultrasound diagnostic techniques. He has also been involved in evoked potential research (depth of anaesthesia) and blood glucose prediction (diabetes therapy). He completed his Ph.D. degree, "Novel strategies for enhancing artificial neural network solutions," in 1996 while lecturing at Strathclyde included work on ECG compression. He has carried out commercial research programs in collaboration with many multinational corporations. Prior to this, he spent 8 years in industry in various companies from startups to multinationals in positions ranging from Graduate Design Engineer to Design Manager. A First Degree in Electronics and Physics from the University of Edinburgh completed in 1983 provided the basis for the eclectic research interests he has now.

Pablo Laguna was born in Jaca (Huesca), Spain, in 1962. He received the Physics degree (M.S.) and the Doctor in Physic Science degree (Ph.D.) from the Science Faculty at the University of Zaragoza, Spain, in 1985 and 1990, respectively. The Ph.D. thesis was developed at the Biomedical Engineering Division of the Institute of Cybernetics (UPC-CSIC) under the direction of Pere Caminal. He is Full Professor

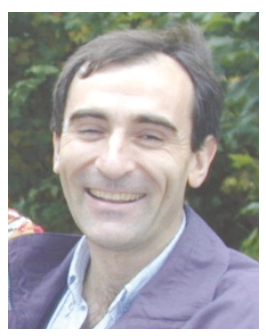
of signal processing and communications in the Department of Electrical Engineering at the Engineering School, and a Researcher at the Aragon Institute for Engineering Research (I3A), both at University of Zaragoza, Spain. From 1992 to 2005, he was Associate professor at the same university, and from 1987 to 1992 he worked as Assistant Professor of automatic control in the Department of Control Engineering at the Politecnic University of Catalonia (UPC), Spain, and as a Researcher at the Biomedical Engineering Division of the Institute of Cybernetics (UPC-CSIC). His professional research interests are in signal processing, in particular applied to biomedical applications. He is, together with L. Sörnmo, the author of Bioelectrical Signal Processing in Cardiac and Neurological Applications (Elsevir, 2005).

Maurice Cohen is Professor of radiology at University of California, San Francisco, and also Professor in the Graduate Groups in biological and medical informatics and the Joint Graduate Group in bioengineering at UCSF and UC Berkeley. He has over 240 publications in the areas of applied mathematics, artificial intelligence in medicine, chaotic modeling, signal analysis, complex systems, neural networks, and image pro-

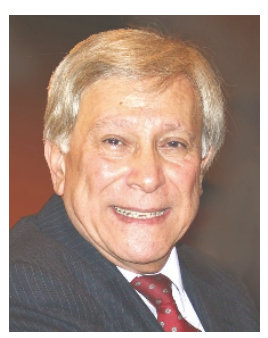
cessing. He has received numerous honors, including the Faculty Research Award from UCSF and Outstanding Professor from CSUF. He was named Renaissance Scholar by the National Honor Society of Phi Kappa Phi. He is a Fellow of the American Institute for Medical and Biological Engineering for his pioneering work in cardiology for which he also was awarded a prize from the American Medical Informatics Association. He solved two problems in mathematics and chaos theory that were believed to be insoluble. In addition, he is an internationally recognized artist and has shown his painting in San Francisco, Carmel, NY, and Paris, including the 2006 Salon of the Sociéte Nationale des Beaux Arts in the Caroussel du Louvre. 